\title{
Electron-donating Effect of Hydantoin Derivative Inhibitor on the Enhanced Anticorrosion Capacity for Mild Steel in Hydrochloric Acid Medium
}

\author{
Junjie Zhu ${ }^{1}$, Hua Hao ${ }^{2, *}$, Xingwen Zheng ${ }^{3}$ \\ ${ }^{1}$ College of Chemistry and Materials Engineering, Beijing Technology and Business University, \\ Beijing 100048, China; \\ ${ }^{2}$ Institute of Chemistry, Chinese Academy of Sciences, Beijing 100190, China; \\ ${ }^{3}$ Key Laboratory of Material Corrosion and Protection of Sichuan Province, Sichuan University of \\ Science \& Engineering, Zigong 643000, China \\ *E-mail: haohuaiccas@126.com
}

Received: 5 November 2021 / Accepted: 22 December 2021 / Published: 2 February 2022

\begin{abstract}
Hydantoin (HDT) and its derivative (allantoin, ALT) were evaluated as corrosion inhibitors for 20\# steel in $1 \mathrm{M} \mathrm{HCl}$ solution at $303 \mathrm{~K}$ through gravimetric test, potentiodynamic polarization, electrochemical impedance spectroscopy and surface analysis. Especially, the electron-donating effect of extra acid diamide group (ALT) on improving the anticorrosion efficiency was observed: the inhibition efficiency of ALT-inhibited specimen (maximum of 96.12\%) was higher than the counterpart protected by HDT at the same dosage. Electrochemical results showed that the corrosion current density was largely suppressed along with the elevated interfacial charge transfer resistance for steel in $\mathrm{HCl}$ solution with ALT. Due to the effective adsorption, both HDT- and ALT-inhibited specimens emerged the inferior surface wettability as compared to that of blank control; surface morphology also evidenced the better protection of ALT than that of HDT. Theoretical modelling indicated that acid diamide group on ALT intensified the negative potential of hydantoin ring, which played a pivotal role in chemisorption and the ensuing corrosion inhibition mechanism for 20\# steel in $\mathrm{HCl}$ solution.
\end{abstract}

Keywords: hydantoin; corrosion inhibitor; electrochemical evaluation; density functional theory; molecular dynamics simulation

\section{FULL TEXT}

(C) 2022 The Authors. Published by ESG (www.electrochemsci.org). This article is an open access article distributed under the terms and conditions of the Creative Commons Attribution license (http://creativecommons.org/licenses/by/4.0/). 